\title{
TU/e emonownen

\section{Recombination kinetics of InAs quantum dots: Role of thermalization in dark states}

\section{Citation for published version (APA):}

Gurioli, M., Vinattieri, A., Zamfirescu, M., Colocci, M., Sanguinetti, S., \& Nötzel, R. (2006). Recombination kinetics of InAs quantum dots: Role of thermalization in dark states. Physical Review B, 73(8), 085302-1/6. [085302]. https://doi.org/10.1103/PhysRevB.73.085302

DOI:

10.1103/PhysRevB.73.085302

Document status and date:

Published: 01/01/2006

\section{Document Version:}

Publisher's PDF, also known as Version of Record (includes final page, issue and volume numbers)

\section{Please check the document version of this publication:}

- A submitted manuscript is the version of the article upon submission and before peer-review. There can be important differences between the submitted version and the official published version of record. People interested in the research are advised to contact the author for the final version of the publication, or visit the $\mathrm{DOI}$ to the publisher's website.

- The final author version and the galley proof are versions of the publication after peer review.

- The final published version features the final layout of the paper including the volume, issue and page numbers.

Link to publication

\section{General rights}

Copyright and moral rights for the publications made accessible in the public portal are retained by the authors and/or other copyright owners and it is a condition of accessing publications that users recognise and abide by the legal requirements associated with these rights.

- Users may download and print one copy of any publication from the public portal for the purpose of private study or research.

- You may not further distribute the material or use it for any profit-making activity or commercial gain

- You may freely distribute the URL identifying the publication in the public portal.

If the publication is distributed under the terms of Article 25fa of the Dutch Copyright Act, indicated by the "Taverne" license above, please follow below link for the End User Agreement:

www.tue.nl/taverne

Take down policy

If you believe that this document breaches copyright please contact us at:

openaccess@tue.nl

providing details and we will investigate your claim. 


\title{
Recombination kinetics of InAs quantum dots: Role of thermalization in dark states
}

\author{
M. Gurioli,* A. Vinattieri, M. Zamfirescu, and M. Colocci \\ CNISM, Department of Physics and LENS, Via Sansone 1, I-50019, Sesto Fiorentino, Italy \\ S. Sanguinetti \\ CNISM, Dipartimento di Scienza dei Materiali, Universita'di Milano Bicocca, Via Cozzi 53, I-20125, Milano, Italy \\ Richard Nötzel \\ eiTT/COBRA Inter-University Research Institute Eindhoven, University of Technology 5600 MB Eindhoven, The Netherlands
}

(Received 27 July 2005; revised manuscript received 21 October 2005; published 1 February 2006)

\begin{abstract}
We present time-resolved photoluminescence measurements of InAs/GaAs quantum dots showing a relevant interplay between recombination kinetics and thermalization processes. By time-resolved and steady-state measurements we experimentally demonstrate that the thermal population of optically inactive states fully accounts for the increase of the intrinsic radiative lifetime in quantum dots when the lattice temperature raises. Experimental data are compared with model predictions for the electronic structure of the quantum dot, and the dark optical transitions involved in the thermalization nicely correspond to the first-hole-excited levels. In addition, the observation of thermalization between the electronic levels allows us to estimate the relaxation time to the ground state, which turns out to be in the ps time scale.
\end{abstract}

DOI: $10.1103 /$ PhysRevB.73.085302

PACS number(s): 78.67.Hc, 78.47.+p

\section{INTRODUCTION}

Self-assembled semiconductor quantum dots (QD's) are currently deeply investigated because of their potentiality as building blocks for novel optoelectronic devices such as nanoemitters and single-photon emitters. Understanding of the carrier recombination dynamics in those nanostructures is therefore a topic of extreme relevance, the performance of the final device being strictly connected to the energy and momentum relaxation kinetics. Carrier relaxation in QD's has been the subject of a long-lasting discussion associated with the prediction of the well-known phonon bottleneck effect, ${ }^{1-3}$ as a consequence of the discrete density of states, which should dramatically reduce the phonon-mediated scattering events. However, it was shown that the phonon bottleneck is not very effective, ${ }^{4-6}$ since several mechanisms such as anharmonic decay of polaron (electron-phonon) (Refs. 4 and 5) and resonant multiphonon processes ${ }^{6}$ can produce a fast energy relaxation, and a convincing picture has finally emerged. Another important issue-i.e., the radiative versus nonradiative recombination - has been analyzed in many details and under different aspects. It is nowadays well established that the thermal quenching of the photoluminescence, observed at high $T$, is due to carrier escape from the $\mathrm{QD}$ to the wetting layer (WL) or barrier materials, where nonradiative recombination occurs. ${ }^{7,8}$ Loss of carriers within the barriers during the relaxation path has been also invoked to explain the thermal quenching at intermediate $T{ }^{9,10}$ However, despite the large effort dedicated to the understanding of the nature of the physical mechanisms underlying carrier relaxation and recombination, some questions are still open: among these, the high-temperature behavior of the intrinsic radiative lifetime, which is of obvious great importance, has not yet been addressed in similar detail with respect to the carrier energy relaxation and nonradiative recombination. In fact contradictory statements from very similar experimental results have been reported in the literature. The increase of the photoluminescence (PL) lifetime of the fundamental optical transition in QD's with increasing temperature has been equally attributed to dark states, ${ }^{11-13}$ to carrier transfer between QD's, ${ }^{14}$ to carrier escape and recapture, ${ }^{15}$ and to reduction of the nonradiative recombination rate. ${ }^{16}$ Clearly a supplement of analysis on this relevant point is needed. In this paper we investigate, by means of PL experiments under different and complementary experimental conditions, the interplay between radiative recombination and thermalization in ruling the recombination dynamics, showing that optically inactive (dark) states play a major role. A careful comparison between time-resolved and steady-state measurements allows us to experimentally prove that the increase with temperature of the intrinsic radiative lifetime in QD's comes from to the thermal population of optically inactive states. By calculating the QD electronic structure within an effective-mass model we conclude that the dark states, which act as a reservoir for the ground state, are associated with the first-holeexcited levels.

\section{EXPERIMENT}

We investigated InAs/GaAs QD's grown by molecular beam epitaxy on a GaAs(100) substrate. After a deposition of a 200 -nm-thick $\mathrm{GaAs}$ buffer layer at $580{ }^{\circ} \mathrm{C}$, the samples were cooled down to $500{ }^{\circ} \mathrm{C}$ for the deposition of 2.0 monolayers (ML) of InAs with a rate of $0.003 \mathrm{ML} / \mathrm{s}$. The QD layer is capped by $100 \mathrm{~nm}$ of GaAs grown at $580{ }^{\circ} \mathrm{C}$. A final deposition of $2.0 \mathrm{ML}$ of InAs at the temperature of $500{ }^{\circ} \mathrm{C}$ is used for atomic force microscopy (AFM) analysis and a QD sheet density of the order of $10^{9} \mathrm{~cm}^{-2}$ has been found. Continuous-wave (cw) PL spectra were measured by using a $\mathrm{Ar}^{+}$laser and a dispersive monochromator operating with an InGaAs photodiode. Time-resolved (TR) PL measurements 


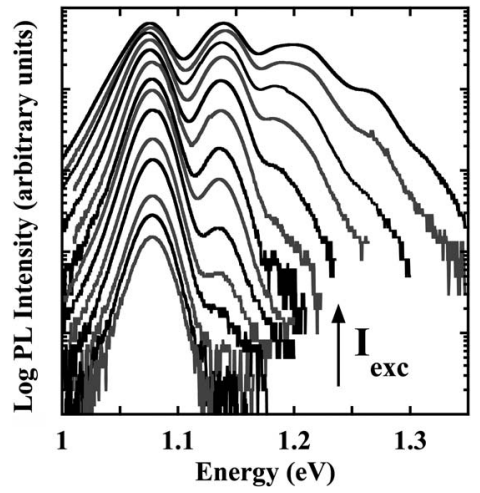

FIG. 1. Typical cw-PL spectra at $10 \mathrm{~K}$ for increasing excitation power density from $0.02 \mathrm{~W} / \mathrm{cm}^{2}$ at step of factor of 2 .

were performed by using a mode-locked Ti:sapphire laser, pumped by a cw $\mathrm{Ar}^{+}$laser, providing 1.2-ps pulses with a repetition rate of $81 \mathrm{MHz}$. The excitation density was changed in the range $0.02-200 \mathrm{~W} / \mathrm{cm}^{2}$. The samples were kept in a closed-cycle cryostat, and experiments were done between 10 and $300 \mathrm{~K}$. The PL was collected and dispersed through a flat-field monochromator with $25 \mathrm{~cm}$ focal length and detected by a InAlAs/InGaAs photomultiplier followed by a photon counting apparatus for PL measurements and a time-correlated single-photon counting setup providing an experimental time resolution (ETR) of 200 ps for TR spectra. An infrared streak camera was also used for high-temporalresolution measurements (4 ps time resolution).

We report in Fig. 1 typical cw spectra at $10 \mathrm{~K}$ for increasing the excitation power density $\left(I_{\text {exc }}\right)$ from $0.02 \mathrm{~W} / \mathrm{cm}^{2}$ at step of factor 2. State filling is observed when increasing the excitation power, and at least four main optical transitions are resolved in the highest-excitation condition. When the excitation density increases the emission from the excited state becomes more important with a superlinear growth. At low $I_{\text {exc }}$ a single PL band peaked at $1.077 \mathrm{eV}$ is found and it corresponds to the QD ground-state (g.s.) optical transition. The first high-energy shoulder at $1.135 \mathrm{eV}$ corresponds to the excited-state (e.s.) recombination from the $p$ shell of the QD's. The inhomogeneous broadenings (at low $I_{e x c}$ ) is of the order of $28 \mathrm{meV}$ denoting a small spread in the QD size.

The ratio $\mathrm{PL}_{\text {e.s. }} / \mathrm{PL}_{\text {g.s. }}$ between the spectrally integrated emission of e.s. and g.s. is reported in Fig. 2(a) as a function of the inverse of thermal energy, for fixed $\mathrm{cw}$ excitation power. Here a slight state filling condition is used in order to follow the e.s. PL band down to low temperature. Up to $100 \mathrm{~K}$ the ratio $\mathrm{PL}_{\text {e.s. }} / \mathrm{PL}_{\text {g.s. }}$ decreases with increasing temperature, denoting a reduction of the state filling - namely, of the number of carriers inside the QD's. This likely follows from the reduction of average number of carriers in the vicinity of the QD's associated with the thermally activated nonradiative channels in the barriers. ${ }^{9} 10$ For temperatures higher than $100 \mathrm{~K}$ the ratio $\mathrm{PL}_{\text {e.s. }} / \mathrm{PL}_{\text {g.s. }}$ increases exponentially with $1 / k T$ denoting the attainment of a thermal equilibrium between the electronic levels involved in the emission. The solid line in Fig. 2(a) refers to the Boltzmann factor expected for an energy splitting of $58 \mathrm{meV}$, and it is in good agreement with the experimental findings. In Fig. 2(b)
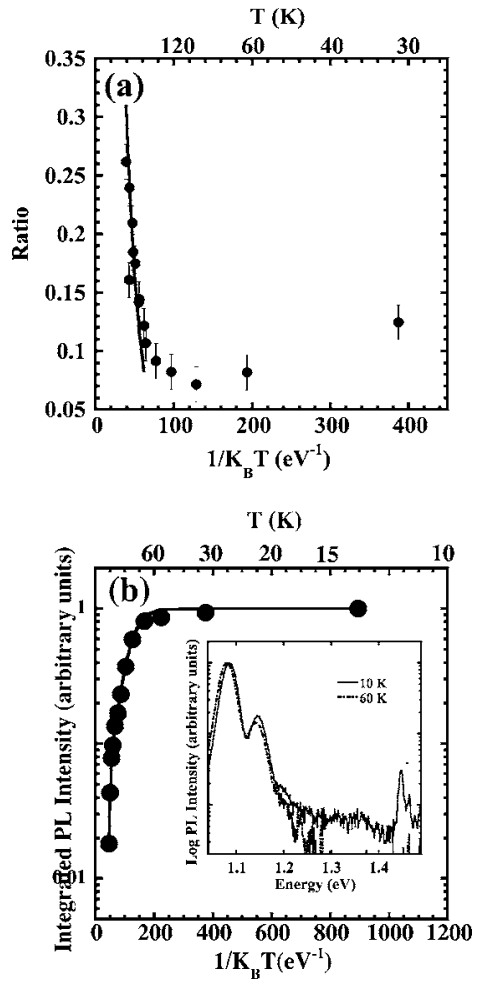

FIG. 2. (a) Ratio $\mathrm{PL}_{\text {e.s. }} / \mathrm{PL}_{\text {g.s. }}$ between the spectrally integrated emission of the e.s. and g.s. as a function of $1 / k T$, for fixed cw excitation power. The solid line refers to the Boltzmann factor expected for an energy splitting of $58 \mathrm{meV}$. (b) Arrhenius plot of the spectrally integrated emission from the QD. The inset shows two cw spectra at different temperatures, indicating the quenching of the emission from the wetting layer. The solid line is the fit of the data performed using the model reported in Ref. 7.

we report the Arrhenius plot of the spectrally integrated cw-PL emission. The inset of Fig. 2(b) shows the cw-PL spectra at two different temperatures in a logarithmic scale; the emission around $1.45 \mathrm{eV}$ comes from the carrier recombination in the WL. Referring to Fig. 2(b), the activation energy associated to the sharp quenching at high temperature (above $220 \mathrm{~K}$ ) nicely corresponds to the energy separation $(380 \mathrm{meV})$ between the QD and the wetting layer optical transitions [see the inset of Fig. 2(b)] in agreement with the thermal escape of carriers out of the QD's. It has to be noted that the escape towards the wetting layer does not produce an increase of the PL from the WL, denoting that the carriers out of the dots mainly recombine nonradiatively, as shown in the smooth quenching observed until $200 \mathrm{~K}$ can be tentatively attributed to thermally activated parasitic channels in the barrier region, according to Refs. 9 and 10.

Typical decays of the TR-PL signal are reported in Fig. 3 (a) at $10 \mathrm{~K}$ for the g.s. and e.s. emission. Here a slight state filling condition $\left(I_{e x c}=1 \mathrm{~W} / \mathrm{cm}^{2}\right)$ is used in order to detect the e.s. PL band at $T=10 \mathrm{~K}$. Also shown, in Fig. 3(a), is the ETR from the time-correlated single-photon counting apparatus: it clearly appears that the full width at half maximum of ETR $(\approx 200 \mathrm{ps})$ does not represent a limit to detect the fast emission from the e.s., with a decay time of $250 \mathrm{ps}$. This value tends to increase for increasing the excitation power 

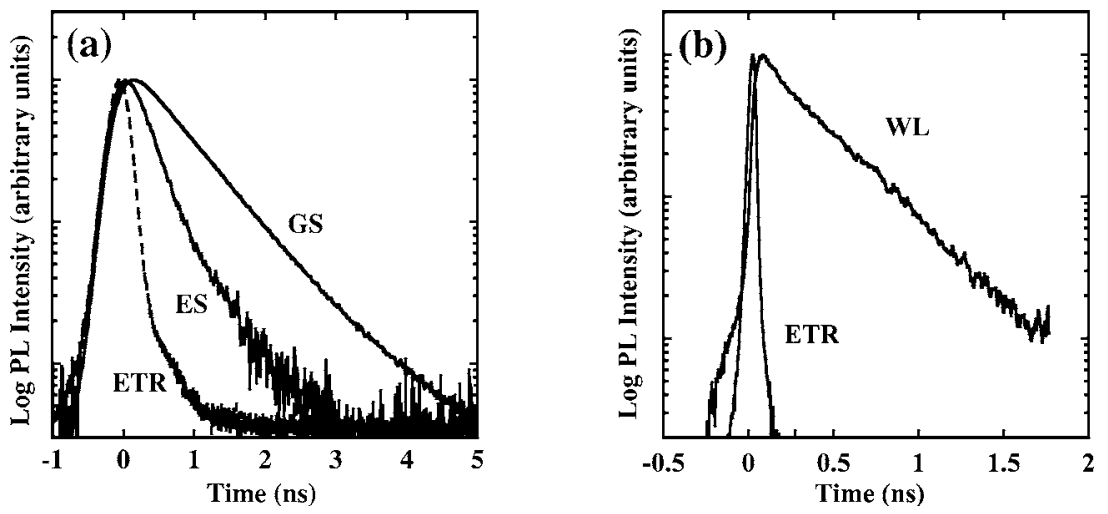

FIG. 3. TR-PL time decays at $T=10 \mathrm{~K}$ for the g.s. and e.s. (b) PL time decay of the WL emission at $10 \mathrm{~K}$. ETR indicates the experimental time resolution. density, likely due to the reduction of the carrier energy relaxation efficiency in state filling condition. Therefore the e.s. lifetime reflects the relaxation rate down to the g.s. and does not represent the intrinsic radiative lifetime. The g.s. lifetime is $700 \mathrm{ps}$, and it does not change when reducing the excitation power over two orders of magnitude. In Fig. 3(b) the PL decay, at $10 \mathrm{~K}$ at the WL energy, is shown, along with the ETR provided by the streak camera detector. A common signature is the fast rise of the PL from the wetting layer and the QD luminescence, which is of the order of a few tens of ps; this is a strong evidence of the lack of relaxation bottleneck, indicating a fast capture in the dots.

In Fig. 4 we report the temperature dependence of the time evolution of the g.s. (solid lines) and e.s. (open circles) recombinations. Increasing the temperature we observe a slowdown of the recombination kinetics: both the PL lifetimes of the g.s. and e.s. increase, eventually assuming the same value, within the experimental errors. The observation of the same temporal profile for the luminescence arising from two different transitions is a strong indication that efficient thermalization occurs also in the pulsed excitation condition. Under thermal equilibrium the relative population of different states depends only on the energy splitting and not on the intrinsic recombination time. This produces an effective recombination time of the whole QD population. Finally for temperatures above $250 \mathrm{~K}$ the drastic reduction of the PL lifetimes shows the dominance of a thermal escape towards the WL, where nonradiative recombination occurs.

\section{DISCUSSION}

The comparison of $\mathrm{cw}$ and time-resolved properties of the g.s. and e.s. recombination allows us to distinguish three different thermal regimes. In the range $10-100 \mathrm{~K}$ no thermalization is found between the populations of the states involved in the g.s. and e.s. optical transitions. Figure 2(a) shows that the ratio between the e.s. and g.s. PL intensity does not follow the Boltzmann factor. In addition the PL decay time of the two optical transitions is very much different. For temperature of the order of $120 \mathrm{~K}$ we observe the onset of thermalization between the population of the states involved in the g.s. and e.s. recombination. This is clearly demonstrated by the significant increase of the ratio between the e.s. and g.s. PL intensity when increasing the temperature, eventually following the expected Boltzmann ratio. As already stated the observation that the temporal profiles of the luminescence arising from e.s. and g.s. optical transitions tend to become the same is a further indication that thermalization is very efficient. At the onset of the thermalization between the electronic states inside the QD, an estimate of the interlevel carrier relaxation time can also be obtained. In fact, thermalization requires that the refilling rate $\Gamma_{u p}$ of the e.s. from the g.s. be larger than the recombination rate $\Gamma_{r e c}$, namely $\Gamma_{u p}>\Gamma_{r e c}$. Then, $\Gamma_{u p}$ can be related to the relaxation rate $\Gamma_{d w}$ from the e.s. down to the g.s., by $\Gamma_{u p}$ $=\Gamma_{d w} \exp (-\Delta E / k T)$, with $\Delta E$ the energy separation of the order of $60 \mathrm{meV}$ between e.s. and g.s. By combining these relations it follows $\Gamma_{d w}>\Gamma_{\text {rec }} \exp (\Delta E / k T)$. Since we observe a thermalization when $T$ is above $120 \mathrm{~K}$ and $\Gamma_{\text {rec }}=1 / \tau_{D}$ is roughly $1.4 \mathrm{~ns}^{-1}$, taking into account the energy separation from the g.s. and e.s. we obtain a lower limit $\Gamma_{d w}$ $>0.5 \mathrm{ps}^{-1}$, proving the lack of phonon bottleneck. This very simple argument also implies that thermal equilibrium between carriers in states separated by a smaller energy splitting would occur at much lower $T$; we will use this statement later on.

The observation of a thermalized emission between g.s. and e.s. recombinations is not in contrast with the slight decrease of the PL intensity observed when increasing $T$, but it only indicates that nonradiative channels, likely in competition with the carrier relaxation path, ${ }^{9,10}$ become effective in this temperature range. Finally for $T$ larger than $240 \mathrm{~K}$ we observe a strong reduction of the PL intensity and lifetime, denoting that the nonradiative recombination is dominant.

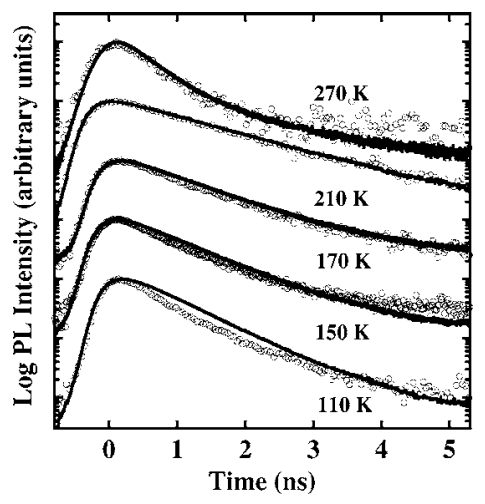

FIG. 4. TR-PL time decays at different temperatures for the g.s. (solid lines) and e.s. (open circles) recombinations. 
From the fit of the PL intensity Arrhenius plot we conclude that, in this temperature regime, the carriers population inside the QD thermalizes with the population in the WL states from where the recombine nonradiatively. The WL acts as a sink, and the carriers promoted via thermalization to the WL states are lost and cannot radiatively recombine in the QD's. Therefore this process is often referred to as carrier escape.

Let us now focus the attention on the origin of the PL lifetime increase with increasing $T$. The pioneering work of Citrin ${ }^{17}$ and Gotoh et al. ${ }^{11}$ showed that, similarly to quantum wells ${ }^{18,19}$ and wires, ${ }^{20,21}$ dark states are predicted in QD's and then their thermal population can lead to an increase of the intrinsic radiative lifetime for increasing $T$. However, as discussed in the Introduction, several further mechanisms could be effective in determining the recombination kinetics in QD's and eventually mask the population related effect. We will now discuss them in detail. The carrier thermal escape from small QD's to the WL and recapture from larger QD's has been invoked in Refs. 14 and 15 as a mechanism for explaining the increase of the PL lifetime with increasing $T$. Our data show that the WL is involved in the carrier recombination kinetics only at very high $T$ (due to the $380 \mathrm{meV}$ activation energy) and, moreover, in this case it acts as a sink for the carrier population. We can therefore exclude the carrier transfer between QD's via the states of the WL. A different picture has been proposed in, Ref. 16, where the increase of the PL decay time with $T$ has been attributed to the reduction of nonradiative rate due to thermal activation of trapped charges that act as recombination centers in the vicinity of QD's. It is worth stressing that this must be accomplished by an increase of the radiative yield in the QD which is in striking contrast with our data. We indeed observe an increase of $\tau_{D}$ in the temperature range where the PL quantum yield drops significantly. In conclusion, our results strongly suggest that the slowdown of the recombination kinetics of carriers in QD's is associated with the increase of the radiative lifetime. In order to get a clear-cut proof of it, we have performed steady-state measurements as a function of $T$ by varying the excitation power in order to keep constant the PL intensity at the g.s. transition of the QD. This latter is proportional to $\Gamma_{R} N_{\text {g.s. }}$, where $\Gamma_{R}$ is the radiative rate and $N_{\text {g.s. }}$ is the population of the ground state of the QD. Therefore a reduction of $\Gamma_{R}$ will produce an increase of $N_{\text {g.s. }}$. Finally, to monitor $N_{\text {g.s. }}$, we set the excitation density to produce a weak state filling condition of the QD at low $T$, so that the possible increase of $N_{\text {g.s. }}$ would produce an increase of excited state filling. Figure 5 reports the spectra obtained in these experimental conditions; the spectra are shifted in energy to compensate for the band gap shrinkage and make the comparison easy. We observe that the state filling condition is almost constant up to $70 \mathrm{~K}$ (not reported in figure); then, it increases for increasing $T$ much more than expected by carrier thermalization. In fact a simple thermalization model, which includes the observed optical transitions and a total number of carriers in the QD to reproduce the low- $T$ PL spectra, predicts, at $T=250 \mathrm{~K}$ and with a constant number of carriers, a filling of the first e.s. which is less than half of the experimental observations. This obviously means that the number of carriers in the QD increases under the condition that $\Gamma_{R} N_{\text {g.s. }}$ stay constant. We believe that this

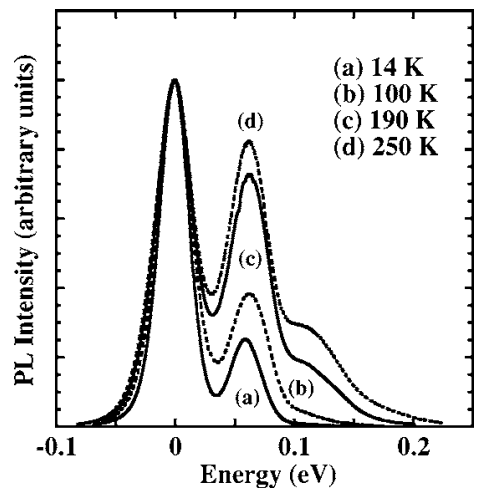

FIG. 5. cw-PL spectra at different temperatures, keeping constant the detected signal from the g.s.

is a striking experimental demonstration of the slowdown of the radiative recombination kinetics in QD's, associated with the thermal population of optical dark states.

Finally, at high temperature the PL lifetime rapidly decreases; this behavior strongly reminds us of the old findings in the quantum well case. ${ }^{22}$ According to our previous discussion and the report of Ref. 22, we interpret this as the insurgency of nonradiative recombination processes associated with carrier escaping out of the QD's, with the rate given by $\Gamma_{N R}(T)=\Gamma_{0} \exp (-\delta / k T)$ where $\delta$ is the activation energy for thermal carrier escape. Obviously the recombination rate will then be given by $\Gamma_{r e c}(T)=\Gamma_{R}(T)+\Gamma_{N R}(T)$, thus explaining the experimentally observed nonmonotonous behavior.

We now address the problem of determining the nature of the dark states, whose carrier population acts as a reservoir for the ground state. Our observations exclude that the wetting layer could act as a reservoir for the QD states; in fact, we have previously shown [see inset of Fig. 2(b)] that nonradiative processes are effective on the WL recombination for $T$ higher than a few tens of kelvin and that escape of the carriers to the WL only occurs above $230 \mathrm{~K}$, when the onset of nonradiative channels is observed from the decrease of $\tau_{D}$. This brings to the conclusion that, if a reservoir of population does exist for the ground state of the quantum dot, such a population is within the QD electronic states. Following the models of Gotoh et al. ${ }^{11}$ the variation of the radiative rate due to the presence of a dark state is given by $\Gamma_{R}(T)$ $=\Gamma_{R}(0) /[1+g \exp (-\Delta E / k T)]$ where $\Delta E$ is the energy difference between the ground state and the dark state and $g$ is the ratio between the degeneracy of the dark state to that of the g.s. In Fig. 6 we compare the experimental data for the g.s. transition and the predictions for different values of $\Delta E$. We want to emphasize that the radiative decay is always expected to be larger than the PL lifetime as suggested by the PL quenching shown in Fig. 2(b) and therefore the theoretical prediction of Fig. 6 represents an upper limit for the PL lifetime. Clearly the thermalization between the observed optical transitions $(\Delta E=58 \mathrm{meV})$ does not fit the experimental data. The theoretical predictions well enough reproduce the experimental data, assuming the presence of a dark state lying at an energy of the order of $10 \mathrm{meV}$ above the carrier g.s. It should be also remarked that the thermalization within the 


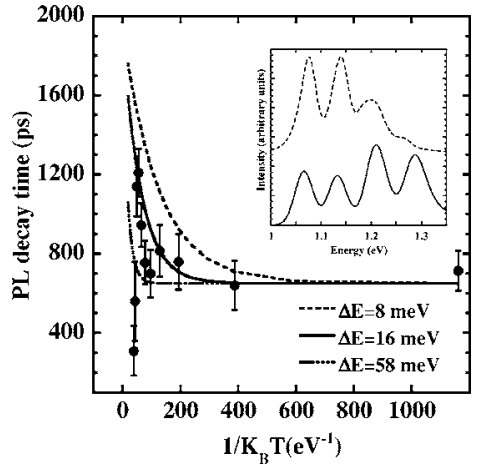

FIG. 6. Comparison between the experimental g.s.-PL lifetime and the predictions for $\Delta E=8 \mathrm{meV}$ (dotted line), $\Delta E=16 \mathrm{meV}$ (solid line), and $\Delta E=58 \mathrm{meV}$ (dashed line). In all cases $g=2$. The inset shows the comparison between the calculated spectrum as discussed in the text (solid line) and the cw experimental spectrum at $10 \mathrm{~K}$ in a band-filling condition (dashed line).

g.s. and this dark state may also start to occur in the lowtemperature regime $(T<100 \mathrm{~K})$, due to the small energy splitting between them. Let us fix the order of magnitude: assuming from our previous estimate $\Gamma_{d w}>0.5 \mathrm{ps}^{-1}$ and $\Delta E=10 \mathrm{meV}$, we find $\Gamma_{u p}>0.05 \mathrm{ps}^{-1}$ already at $T=50 \mathrm{~K}$. It is therefore expected that the model of Gotoh et al. ${ }^{11}$ will be valid down to the low-temperature regime.

The value of the estimated position of the energy level for the dark state suggests that holes, which have higher effective-mass than electrons, are involved in the optically inactive transitions. In order to check this statement we have used an effective-mass model assuming the QD to have a cylindrical symmetry and a dome shape; details of the model can be found elsewhere. ${ }^{23}$ Besides the level energies we also evaluate the electron-hole overlap for any possible $e-h$ transition. The inset of Fig. 6 shows the cw-PL spectrum at $T$ $=10 \mathrm{~K}$ (dashed line) under the strong state filling condition, where four optical transitions are resolved. We therefore adjust the QD size in order to fit the main transition energies. The parameters used in the calculations are reported in Table I. Effective-mass and confinement-potential values were taken from Ref. 24. The solid line in the inset of Fig. 6 gives the absorption spectra predicted by the model using a 30-meV Gaussian broadening for each transition, according to the experimentally measured broadening. The model predictions nicely fit the experimental data, allowing us to obtain information on the QD electronic structure. It is impor-
TABLE I. Parameters used in the effective-mass calculations: $m_{B}$ barrier region effective mass (in electron mass units $m_{0}$ ) $m_{W}$, QD region effective mass (in $m_{0}$ ); $V_{0}$, carrier confining potential (in $\mathrm{meV}) ; H$, QD height (nm); $D$, QD diameter (nm).

\begin{tabular}{|c|c|c|c|c|c|c|c|}
\hline \multicolumn{3}{|c|}{ Electron } & \multicolumn{3}{|c|}{ Hole } & \multirow[b]{2}{*}{$H$} & \multirow[b]{2}{*}{$D$} \\
\hline$m_{B}$ & $m_{W}$ & $V_{0}$ & $m_{B}$ & $m_{W}$ & $V_{0}$ & & \\
\hline 0.0665 & 0.040 & 450 & 0.3774 & 0.59 & 266 & 3.8 & 27.0 \\
\hline
\end{tabular}

tant to note that the model predicts many electron and hole states. Due to the differences in the effective masses, we found that the splitting between electron (hole) levels is of the order of $50 \mathrm{meV}(8 \mathrm{meV})$. Beside the large variety of optical bright transitions reported in the inset, there are several optically inactive transitions. In particular we found that the first two hole excited levels (at 8 and $16 \mathrm{meV}$ with respect to the hole ground state) are dark with respect to the fundamental electron transition. We therefore conclude that they will act as dark states for the electrons populating the fundamental level.

\section{CONCLUSIONS}

By means of PL measurements under different experimental conditions we have demonstrated that the increase of the radiative lifetime in InAs QD's is due to the thermal population in excited states of the QD. A comparison with a simple effective-mass model suggests that the reservoir of population comes from excited hole states which correspond to dark optical transitions. The strong interplay between radiative processes and thermalization in optically inactive states fully accounts for the observed increase of the PL decay time. Moreover, our data provide clear evidence of the lack of relaxation bottleneck and of an efficient carrier capture in the quantum dot. We believe that the correct knowledge of the high-temperature behavior of the intrinsic radiative lifetime is of the utmost importance for improving QD optoelectronic device performance.

\section{ACKNOWLEDGMENTS}

M.Z. acknowledges financial support from European Contract No. MRTN-CT-2003-503677. The authors acknowledge support by the National Project FIRB-Nanoelettronica. Work at LENS is supported by the EC under Contract No. HPRICT 1999-00111.
*Corresponding author. Electronic address: gurioli@lens.unifi.it

${ }^{1}$ H. Benisty, C. M. Sotomayor-Torres, and C. Weisbuch, Phys. Rev. B 44, 10945 (1991).

${ }^{2}$ R. Heitz, H. Born, F. Guffarth, O. Stier, A. Schliwa, A. Hoffmann, and D. Bimberg, Phys. Rev. B 64, 241305(R) (2001).

${ }^{3}$ J. Urayama, T. B. Norris, J. Singh, and P. Bhattacharya, Phys. Rev. Lett. 86, 4930 (2001).

${ }^{4}$ O. Verzelen, R. Ferreira, and G. Bastard, Phys. Rev. Lett. 88,
146803 (2002)

${ }^{5}$ O. Verzelen, G. Bastard, and R. Ferreira, Phys. Rev. B 66, 081308(R) (2002).

${ }^{6}$ M. I. Vasilevskiy, E. V. Anda, and S. S. Makler, Phys. Rev. B 70, 035318 (2004).

${ }^{7}$ S. Sanguinetti, M. Henini, M. Grassi Alessi, M. Capizzi, P. Frigeri, and S. Franchi, Phys. Rev. B 60, 8276 (1999).

${ }^{8}$ E. C. Le Ru, J. Fack, and R. Murray, Phys. Rev. B 67, 245318 
(2003).

${ }^{9}$ K. Mukai, N. Ohtsuka, and M. Sugawara, Appl. Phys. Lett. 70, 2416 (1997).

${ }^{10}$ D. Colombo, S. Sanguinetti, E. Grilli, M. Guzzi, L. Martinelli, M. Gurioli, P. Frigeri, G. Trevisi, and S. Franchi, J. Appl. Phys. 94, 6513 (2003).

${ }^{11}$ H. Gotoh, H. Ando, and T. Takagahara, J. Appl. Phys. 81, 1785 (1997).

${ }^{12}$ A. Fiore, P. Borri, W. Langbein, J. M. Hvam, U. Oesterle, R. Houdré, R. P. Stanley, and M. Ilegems, Appl. Phys. Lett. 76, 3430 (2000).

${ }^{13}$ X. Chen, P. Dawson, M. J. Godfrey, and M. Hopkinson, Phys. Status Solidi C 224, 107 (2001).

${ }^{14}$ A. Melliti, M. Maaref, F. Hassen, M. Hjiri, H. Maaref, J. Tignon, and B. Sermage, Solid State Commun. 128, 213 (2003).

${ }^{15}$ H. Yu, S. Lycett, C. Roberts, and R. Murray, Appl. Phys. Lett. 69, 4087 (1996).
${ }^{16}$ E. Dekel, D. Regelman, D. Gershoni, E. Ehrenfreund, W. Schoenfeld, and P. Petroff, Solid State Commun. 117, 395 (2001).

${ }^{17}$ D. Citrin, Superlattices Microstruct. 13, 303 (1993).

${ }^{18}$ J. Feldmann, G. Peter, E. O. Göbel, P. Dawson, K. Moore, C. Foxon, and R. J. Elliott, Phys. Rev. Lett. 59, 2337 (1987).

${ }^{19}$ M. Gurioli, A. Vinattieri, M. Colocci, C. Deparis, J. Massies, G. Neu, A. Bosacchi, and S. Franchi, Phys. Rev. B 44, 3115 (1991).

${ }^{20}$ D. Y. Oberli, M. A. Dupertuis, F. Reinhardt, and E. Kapon, Phys. Rev. B 59, 2910 (1999).

${ }^{21}$ A. Feltrin, J. L. Staehli, B. Deveaud, and V. Savona, Phys. Rev. B 69, 233309 (2004).

${ }^{22}$ M. Gurioli, J. Martinez-Pastor, M. Colocci, C. Deparis, B. Chastaingt, and J. Massies, Phys. Rev. B 46, 6922 (1992).

${ }^{23}$ J. Y. Marzin and G. Bastard, Solid State Commun. 92, 437 (1994).

${ }^{24}$ M. Califano and P. Harrison, Phys. Rev. B 61, 10959 (2000). 